\title{
EDUCACIÓN MEDIA TÉCNICO PROFESIONAL EN TURISMO: IMAGINARIOSOCIAL, MOTIVACIÓN Y AGENTES INTERVINIENTES EN LA ELECCIÓN, EXPECTATIVAS Y PROYECCIONES. ESTUDIO DE CASO, INSTITUTO SUPERIOR DE ADMINISTRACIÓN Y TURISMO, VALDIVIA
}

\author{
Mauricio Triviños \\ Universidad Austral de Chile
}

\section{RESUMEN}

Esta investigación está basada en el estudio de caso del Liceo de Educación Media Técnico Profesional, de ahora en adelante, (EMTP), llamado Instituto Superior de Administración y Turismo, de ahora en adelante (INSAT), de la ciudad de Valdivia, Chile, y es parte de una indagación mayor, enmarcado en una tesis de magister. Con este estudio se busca la conformación del imaginario Social vinculado a la elección de la modalidad de educación Media Técnico Profesional en Turismo.

Como eje central del estudio de caso, se trabajó de forma específica con los estudiantes de 3er. año de la especialidad de Servicios Turísticos del establecimiento mencionado. La construcción de este imaginario se basa para esta investigación en cuatro aristas vinculadas al momento decisional, como lo son: Motivación y Agentes Intervinientes en la elección, Expectativas y Proyecciones.

El trabajo metodológicamente se desarrolla con una batería de herramientas y cruce de información entre los actores considerados relevantes en este estudio de caso, como lo son: Estudiantes, Profesores, Directivos de establecimientos, Agentes de la gobernanza del sistema educativo público a nivel regional, Padres y Agentes del mercado.

Ante la necesidad de intentar analizar el fenómeno de la EMTP, se hace gravitante entender sus dinámicas desde un sentido amplio, dada la complejidad, diversidad y multisectorialidad del fenómeno o modalidad. Ante lo cual, no es menor la trascendencia que ha alcanzado dicha modalidad, más si tomamos en cuenta que en la actualidad casi la mitad de los alumnos de la enseñanza media de nuestro país lo hace bajo este formato de educación secundaria, que suma y suma alumnos de forma constante, muchas veces debido a que sus familias ven en ella una buena oportunidad de desarrollo y estabilidad laboral y económica.

Palabras claves:

Imaginario Social, Turismo; Técnico profesional, Elección y Expectativas 


\title{
TECHNOLOGICAL EDUCATION IN TOURISM: SOCIAL IMAGINARY, MOTIVATION AND AGENTS INTERVENING IN THE STUDENT'S DECISION. CUALITATIVE CASE STUDY OF INSTITUTO SUPERIOR DE ADMINISTRACIÓN Y TURISMO, VALDIVIA
}

\author{
Mauricio Triviños \\ Universidad Austral de Chile
}

\begin{abstract}
This research aims to examine the social imaginary construction by students that choose to study tourism at technological schools. Four key aspects emerging from the literature are examined to investigate this: motivation of the students, agents intervening in the student's decision, expectative and projections.

A qualitative case study was adopted to investigate this. 13 students were indepth interviewed from a polytechnic school in Valdivia (Chile), whose specialization is in the area of tourism management (Instituto Superior de Administracion y Turismo - INSAT). Data was also gathered from the principal of the school, teachers, parents and public and private stakeholders related to the regional education sector. In these cases questionnaires and interviews were also conducted. Participatory observation in classrooms was also carried out to examine behaviour of the students and relationships with their teacher.
\end{abstract}

Although this research is still in process, preliminary results show three key aspects. First, students primarily choose polytechnic schools based on their motivation of achieving specific skills that allow them to get a job after finishing school. Second, regarding the agents intervening in the students' decision, $100 \%$ of the students indicated that the decision of picking up these schools is basically from themselves. However, additional data shows that parents and family group strongly influence the students' final decision. Finally, preliminary data also shows that just a part of the sample is interested in follow studies regarding their specialization at the professional level (i.e. in Universities).

Keywords:

Social imaginary, tourism, Polytechnic, Choose and expectations 


\section{MARCO TEORICO}

"Las reformas de los sistemas de educación técnica y profesional deben Explorar el cómo, con qué instrumentos, mediante qué medidas, a través de qué soluciones, de acuerdo con qué enfoques y estrategias deben implementarse estas reformas" (Blas, 2009, pág. 160).

Durante su historia y de forma graduada la EMTP ha buscado entregar una orientación práctica a la educación chilena, esto se acentúa a medida que las ideas pedagógicas han realzado el aprendizaje por medio de la experiencia. Otro punto de inflexión que acentúa la relevancia de la EMTP es la situación y esquema económico que de una u otra forma también contribuye al potenciamiento de este modelo educativo, ya que la constante necesidad de incluir a los llamados mandos medios o personal operativo realza la importancia de los egresados y titulados de la tan popular EMTP. Esto según lo mencionado entre otros por Castro, E., y Orellana, W. (2010).

\section{La Educación en la Naciente República.}

Entre los primeros pasos hacia la instauración institucional del sistema educativo chileno, destacan los dados por el presidente M. Bulnes al promulgar la ley que permite la creación de la Universidad de Chile, para de esta forma contribuir al desarrollo cultural y social de la nación, proporcionando con esta acción, la creación de un marco para el futuro desarrollo educacional, sin embargo, y considerando la importancia de estos pasos debieron pasar años para el fortalecimiento de un sistema técnico de enseñanza.

Para autores como Miranda, M. (2003), y Sepúlveda, L., Ugalde, P., \& Campos, F. (2008), con los aconteceres que rodeaban a la floreciente nación, aún no se observa algún claro de luz convincente, consensuado y mancomunado por parte de los gobernantes para instaurar, o más bien, poner sobre la palestra nacional la EMTP. Es recién en las cercanías del 1850 que de la mano de la llamada Sociedad de la Igualdad, que comienza a ver la luz la educación técnica profesional en Chile, es así como esta sociedad paralelamente contemplaba la creación de escuelas gratuitas, baños públicos, bancos de obreros, montes de piedad y otros.

En directa relación con la educación técnica, es hacia el 1849 cuando se dan pasos de relevancia nacional con la creación de la Escuela de Artes y Oficios de Santiago y la escuela de Bellas Artes y Arquitectura. Tomando el impulso dado por la creación de estas casas de estudio y en apertura social hacia los derechos de las mujeres es que se funda la Escuela Técnica Femenina de Santiago. A partir de la creación de estas primeras instituciones más tarde se crearon una serie de institutos y escuelas prácticas a lo largo de nuestro país, que de una u otra forma tenían relación con la educación técnico profesional. 
Manuel Salas fue el primero en realizar acciones concretas para desarrollar en la nación una educación "Científica y Práctica", trabajando arduamente para la creación e instauración de la reconocida Academia de San Luis en 1897, es en esta línea que destaca lo señalado por Pino que nos indica, "no sólo fundó nuestra educación técnica de nivel medio, sino también, aplicó por primera vez en nuestro país un método racional, científico". La Academia fue concebida como "una respuesta a la realidad socioeconómica y cultural que, habiéndola estudiado acuciosamente, era el más certero diagnóstico y el plan más visionario para impulsar el desarrollo de Chile" (Pino, 2003, p. 73). Si bien esta academia no es el inicio oficial ni formal de la EMTP podemos señalar que actúa como una base o bien es un punto de partida.

\section{Época de Cambios y Modelos de la EMTP}

Los inicios del siglo XIX, se convirtió en una época de cambios que contribuyeron, sin duda al establecimiento del modelo educacional de la EMTP. Como señalan Sepúlveda, L., Ugalde, P., \& Campos, F. (2008), Brunner, J. (2000), entre otros, los cambios sociales y políticos de la época, como lo fueron, la fundación del Partido Obrero Socialista de Chile que a la postre se transformaría en el Partido Comunista o la inclusión de capitales estadounidense en el yacimiento de Chuquicamata en el año 1913 y así como lo fue el inicio de la Primera Guerra Mundial, transforman a esta época en un ciclo de cambios que propiciaron una transformación de la nación. Es en base a estos y otros cambios que comienza a hacerse palpable el desarrollo y la importancia del sistema educacional y en específico la educación técnica profesional. Es así como en 1915 se promulga el decreto que crea la Comisión de Enseñanza Comercial con la idea de velar por el desarrollo de esta modalidad educacional, pasados algunos años y bajo la misma primicia de la anterior, se crea el consejo de Enseñanza Industrial de Hombres, y así sucesivamente se fueron creando distintas comisiones y direcciones relacionadas con el quehacer productivo nacional, como la agricultura, la ganadería, el comercio y la artesanía.

En 1960 la Dirección General de Educación cambia de nombre y pasa a llamarse "Dirección de Educación Profesional" con funciones en el mundo Técnico Profesional, tales como supervisar la actividad y mantener vínculos con egresados y empresas. A partir de estos cambios se fija el Reglamento General de la Educación Técnico Profesional en el año 1962.

Es en el Decreto ley № 220 del 18 de Mayo de 1998 que se establece claramente los objetivos de la educación Profesional y se deja explicito su relación con el mundo productivo y su vínculo con el desarrollo social y económico del país. "Formar Recursos Humanos calificados de nivel medio, para atender a las áreas de servicios y productivas que requiere el desarrollo socioeconómico del pais" La EMTP en nuestro 
país está compuesta en su estructura básica por cinco ramas: (Comercial; Industrial; Técnica; Agrícola y Marítima) esta ley establece objetivos fundamentales y contenidos mínimos obligatorios para enseñanza media y fija normas generales para su aplicación, esto según (MINEDUC, 1998)

En nuestro país existe un amplio número de establecimientos Técnico Profesionales que aportan claramente al conocimiento específico de un sin fín de actividades productivas, las cuales no hacen otra cosa que preparar la maquinaria que debiera mover al pais. Según MINEDUC. "Los lineamientos de política de enseñanza media está orientada a mejorar la calidad de la educación para todas y todos los jóvenes estudiantes, busca alcanzar mejores aprendizajes, expresados en trayectorias educativas que permitan su progreso para la continuidad de estudios y la adecuada inserción social, ciudadana y laboral” DIPLAP - MINEDUC.

Los establecimientos de EMTP se rigen por D.F.L. $\mathrm{N}^{\circ} 2$; también existen los dependientes de corporaciones privadas los cuales en forma particular se rigen por el D.L. $\mathrm{N}^{\circ} 3.166$, es importante mencionar que, si bien hay especializaciones donde existe un predominancia de género por razones culturales, sociales o bien laborales, no obstante lo anterior, no existe distinción de género en el ingreso y matrículas de estos establecimientos, pudiendo en ellos ingresar de igual forma tanto hombres como mujeres.

Es relevante lo señalado por Castro quien indica en relación con los objetivos de la EMTP actual, "El objetivo era diseñar una formación técnica amplia y flexible, más acorde con las estructuras laborales postfordistas que emergían en el pais, lo que se oponía al modelo imperante que se basaba en entregar una formación muy especializada que buscaba un ingreso temprano al trabajo" (Castro \& Orellana, 2010).

\section{Valoración de la EMTP}

La valorización de la EMTP es múltiple y variada. Se dice, desde que es el motor que mueve a un país en desarrollo, hasta la constante frase que menciona, que un país en desarrollo necesita mayor cantidad de técnicos en desmedro de los profesionales. A esto le podemos sumar que según el coordinador de Formación Técnica del Programa Chile Califica de los ministerios de Economía, Educación y Trabajo, Martín Miranda, "hoy los estudiantes y las familias evalúan la EMTP, como una educación más motivante y significativa y porque además posibilita la inmediata inserción laboral de los estudiantes".

Sumándose a la importancia que sabemos tiene en un país la EMTP es necesario que esta sea oportuna, pertinente y de calidad. Por ende cualquier sea la intervención, o bien, 
propuesta que en nombre de ella se haga, debe incluir a todos los actores intervinientes en ellas. Si bien la problemática de la EMTP ha existido siempre, está a mudado, ha cambiado de tópico, por ende si en el siglo pasado fue la cantidad de establecimientos como señala Véliz "Así como en este país de agricultores sólo hubo siete escuelas fiscales que formaran jóvenes para ser más eficientes en tareas del campo. En este país de mineros, en 1909, sólo existían tres Escuelas Prácticas de Minería". (Véliz, 1965) hoy en día deja de ser el problema la cobertura y se muda hacia otros vectores, como la pertinencia o la claridad, entre otros.

\section{Desarrollo Cognitivo y Decisión}

Los establecimeintos de EMTP que existen en nuestra región, aportan claramente al conocimiento específico de un sin fín de actividades productivas relacionadas con el quehacer local, este conocimiento especifico, si bien es altamente necesario, podriamos decir que a su vez es altamente "racionalizador" del saber, ya que a tan temprana edad actúa como un conjunto cerrado en el cual solo existe un acotado y definido número de componentes funcionales y afines con el "saber hacer" que se saltan u omiten el "saber y el ser". Es delicado pensar que menores de 15 años pertenecientes en la mayoria de los casos a los quintiles más bajo de la sociedad, con altos grados de vulnerabilidad, tenga que elegir su futuro laboral a tan temprana edad, en contraste con otros que lo hacen a partir de los 18 años he incluso más tarde cursando bachilleratos en los primeros años de unversidad. Consirerando para ello lo que mencionan Durkheim, E. (2000) y Feldman, R. (2006), entre otros.

Si bien no se desconoce el aporte realizado por los establecimientos de EMTP que en muchos casos son el único punto visible para intentar salir del entorno que los rodea, es desventajoso, por decirlo menos, someterlos a un momento decisional de tanta relevancia para ellos y que supone un nivel de alta tensión, ante lo cual es válido preguntarse: ¿Qué?, ¿Por qué?, ¿Para quién? y ¿Para que? están decidiendo ellos. Tanto es el trasfondo de la decisión adolescente sobre una profesión o futuro laboral que no es menor exponer lo descrito por Feldman, que en un estracto menciona la diversidad de pensamientos, actitudes y situaciones que convergen en un individuo (adolescente) que llevarlos a pensar en una decisión tan gravitante parece desmesurado.

"Aunque un grupo de chicos no se conocen, comparten angustias comunes de la adolescencia: inquietudes relacionadas con amigos, padres, apariencia, independencia y su futuro... la adolescencia es un periodo crucial. Se trata de un lapso con cambios profundos y en ocaciones confusión. Se suceden cambios biológicos considerables cuando los adolescentes alcanzan la madurez sexual y física. Al mismo tiempo, y, rivales de estos cambios, ocurren importantes modificaciones sociales, emocionales y cognitivas cuando los adolescentes luchan por independencias y se encaminan a la 
edad adulta" (Feldman, 2006).

\section{Desarrollo Psicosocial y Decisión.}

Sumando antecedentes para la investigación y el cuestionamiento del mismo, es válido mencionar otro punto de relevancia para el desarrollo del adolescente y su momento decisional, como lo es el desarrollo cognitivo del individuo. Sabido es que es muy distinto el desarrollo cognitivo de un pre-adolescente, un adolescente y un adulto. Por ejemplo, para el psicólogo Lawrence Kohlberg, los individuos pasan por una serie de etapas de evolución de su sentido de justicia, y por ende del tipo de razonamiento por el cual realizan juicios o bien toman decisiones, según Kohlberg "los pre-adolescentes suelen pensar en términos de reglas concretas e invariables (robar siempre está mal o me castigarían si robara) o en términos de reglas de la sociedad (las personas buenas no roban o ¿Qué pasaría si todos robaran?). (Kohlberg, 1984). Por ende un menor de 15 años, está en medio de su desarrollo cognitivo, aún no ha alcanzado su nivel pleno, si bien esta teoría del comportamiento moral habla de tres niveles de moralidad para un individuo, está, tiene matices y ejerce una elevada influencia en este tema. Pero aun así Kohlberg señala que un grupo minoritario de adultos alcanza el nivel 3 "Nivel 3 de Moralidad: Moralidad pos-convencional, en este nivel, las personas emplean principios morales que se consideran más generales que los de cualquier sociedad en particular" (Kohlberg, 1984).

No es raro en la adolescencia preguntarse ¿Quién soy?, ¿Cómo encajo en el mundo? o bien ¿De qué se trata la vida? Es en esta etapa donde los jóvenes intentan encontrar un lugar en la sociedad, búsqueda que en muchos casos no tiene claro su fecha de término. Esta búsqueda claramente los puede llevar en varios caminos en los cuales debieran tener retorno en este periodo de exploración. Según la teoría del desarrollo psicosocial de Erikson, se destaca la búsqueda constante de la identidad durante la adolescencia, el desarrollo psicosocial implica el cambio de la comprensión que las personas tienen de sí misma, de los demás y del mundo que los rodea.

\section{Imaginario Social.}

La imaginación es el origen de lo que puede ser figurado, pensado, representado, deseado y en relación a lo cual se despliegan los afectos y la construcción de nuestro entorno y "realidad". En el imaginario podemos identificar dos dominios incoercibles, de los cuales nacen dos fuentes del mismo:

- $\quad$ La psique, de la cual se desprende la imaginación radical.

- $\quad$ El dominio histórico social, del cual se desprende el imaginario social.

Desde la imaginación radical se observa la capacidad de la psique de construir incesantes representaciones, intenciones, deseos y afectos, que se producen una seguridad, que no 
está en lugar de nada, ni es delegada de nadie. Es radical porque alude a la raíz, a la fuente de la creación. Para la psique lo que "es", es producido por la imaginación radical. Desde el dominio histórico-social sobresale el imaginario social y su construcción colectiva, pero desde el individuo, por medio de la construcción y desarrollo de éste imaginario social se constituyen las producciones de sentido, sistemas de significación social, por medio del cual se mantiene cohesionada a la sociedad, para lo cual es vital la institución de normas, valores y concepciones que hacen que una sociedad sea visualizada como una unidad. Dentro del imaginario social, Castoriadis distingue dos dimensiones: el imaginario radical y el imaginario efectivo. El imaginario radical "es la capacidad de hacer surgir como imagen algo que no es" (Castoriadis, 1983, pág. 220).

\section{Objetivos.}

\section{a. Objetivos Generales}

Interpretar y construir el imaginario de los estudiantes de la modalidad de EMTP en relación a las motivaciones, expectativas, proyecciones y campo ocupacional, al momento de optar por esta modalidad de estudios, desde el discurso de los actores.

\section{b. Objetivos Específicos.}

- Categorizar cuáles son las motivaciones y expectativas que tienen los estudiantes de la EMTP con la elección de esta modalidad y la configuración que adopta el imaginario social en este proceso de elección.

- Caracterizar las principales proyecciones y campos ocupacionales de los estudiantes de la modalidad EMTP y su construcción por medio del imaginario social.

- Analizar y categorizar los momentos decisionales y agentes interventores, que contribuyen a la elección de los estudiantes sobre la modalidad EMTP y una posterior especialización, estableciendo la o las acciones que ejercen los agentes, tantos internos como externos en este proceso.

- Describir la especificidad de las creaciones de sentido de los estudiantes pertenecientes a la modalidad EMTP en torno a la educación y el ámbito laboralempresarial. 


\section{Metodología}

\section{a. Lineamientos Metodológicos.}

Estos deben ser acordes y coherentes con las premisas asumidas desde el momento en el cual se posiciona al imaginario histórico-social y discurso como la línea transversal del desarrollo y análisis de los participantes de este caso de estudio. En estos lineamientos metodológicos se debe asumir una continuidad y convergencia entre aquellas y los lineamientos epistemológicos, trabajando desde la perspectiva foucaultiana la convergencia y continuidad entre la metodología y la epistemología. Dentro de los principales lineamiento metodológicos que sostienen al presente estudio podemos señalar:

- La búsqueda de un análisis no representacionista: Por medio de este método se busca dar cuenta de lo real, de manera emancipada del investigador y el discurso que tenga cargado en su disco duro creando con ello una institución del imaginario social. Según Castoriadis. "no hay, pues, ni metodología en sentido estricto, ni posibilidad de demostrar rigurosamente algo" (Castoriadis, 2004 pág. 34). Esto en relación al imaginario histórico-social.

- $\quad$ El sujeto como función del discurso: Para Castoriadis, si bien no hay ningún medio o método para encontrar lo que es el núcleo de lo verdadero, e incluso si se encuentra, nunca podría demostrarse que lo es, para el caso a investigar y para cualquier indagación que se realice independiente de la línea metodológica nunca podremos obtener la verdad absoluta y siempre debemos considerar que es la visión de uno o más sujetos (Castoriadis, 2004).

- La caja de herramientas y su funcionalidad: Para la construcción de esta investigación se contempla un análisis del discurso, ante ello es necesario incluir y analizar los lineamientos desarrollados por Foucault, quien se diferencia de los analistas tradicionales del discurso por medio de la concepción y significancia que este hace de lo que se entiende por discurso. Los cuatro principios básicos de la Caja de herramientas según Foucault:

- Principio de Trastocamiento.

- Principio de Discontinuidad.

- Principio de Especificidad.

- Principio de Exterioridad. (Foucault, 2005) 


\section{b. Diseño Metodológico}

Según los objetivos planteados para la presente investigación y las implicancias sociales que en ella se alcanzan, se ha optado por un diseño metodológico trasversal a las visiones tanto micro como macro del fenómeno social a investigar como lo es la EMTP, específicamente la relacionada con el eje productivo del turismo. Para lo cual se ha considerado, por una parte, la aplicación de entrevistas a sujetos con una heterogeneidad de pensamientos y discurso, de modo de encarnar a las distintas voces que intervienen en el mundo educacional en el cual se intervendrá y que construyen de una u otra forma el imaginario social entorno a la EMTP.

A modo de profundización y triangulación se incluirá el análisis de documentación formal, institucional y oficial que delimitan las políticas relacionadas con la EMTP, con esto se hace alusión particularmente aquellos documentos provenientes de organismos internacionales pertinentes, del Ministerio de Educación de Chile, el programa Chile Califica y de la institución en la cual se realizará la investigación.

\section{- Rapport:}

Es vital para el éxito de esta intervención efectuar un adecuado contacto previo o acercamiento con las partes y su ambiente, de modo de no actuar como un agente externo al cual hay que entregarle información sin saber ¿para qué?, ni ¿Por qué?. Se busca en primera instancia con contactos que faciliten los procesos posteriores tanto con los sujetos usuarios de la modalidad, como con el gobierno, o bien, los entes jerárquicos del mismo. Para esta investigación se establece como contacto previo una línea de tiempo de 1 mes en el cual se realizarán para todos los grupos las siguientes actividades:

- Presentaciones formales del proyecto.

- Acercamiento con los posibles entrevistados.

- Entrega de carta Gantt.

- Presentación de los instrumentos.

- Discusión de los resultados esperados con la aplicación de los instrumentos.

\section{- Observación:}

En este apartado se busca principalmente consolidar los lazos creados con el rapport y descubrir los nudos emergentes del discurso que no han sido establecidos anteriormente. 


\section{- $\quad$ Entrevista:}

Utilizar las entrevistas como un eje metodológico, se debe principalmente a los objetivos planteados, específicamente al objetivo general que dice relación con: Realizar un diagnóstico tendiente a identificar la subjetividad de las motivaciones, expectativas, las proyecciones y campo ocupacional de los estudiantes de la modalidad de EMTP a través de la construcción del imaginario social en torno a esta modalidad. En esta línea, los objetivos de forma transversal buscan indagar en las percepciones, expectativas y proyecciones del alumnado de la modalidad de EMTP, por medio de estas se busca relevar el vínculo existente entre la subjetividad, las políticas y las contingencias educacionales, para de esta forma dar cuenta de un diagnóstico por medio del imaginario social.

A su vez al estar inmerso en el mundo de la EMTP, en particular en el caso del establecimiento INSAT, implica entender y aceptar relaciones de poder, donde por ende uno de sus objetos es la subjetividad y es en ellos donde el poder y las pugnas por el mismo nacen y se llevan a la sociedad "real". Es más que seguro que un joven de 14 o 15 años no haya leído en su vida un texto oficial relacionados con las políticas de la modalidad EMTP o de la visión-misión de su establecimiento, pero claramente vive y convive con estas políticas.

\section{Criterios para la elección de los sujetos:}

Según lo expuesto con anterioridad en relación a la construcción del imaginario y considerando que este por ningún motivo pretende establecer verdades absolutas, ni líneas representativas de esta realidad, al establecer los criterios de selección de los sujetos para las entrevistas, se debe dejar en claro que estos no responden a una búsqueda de objetividad, ni de la neutralidad de otros estudios, porque estos desde la perspectiva epistemológica de la metodología expuesta sería una mentira y de una contrariedad difícil de salvar y por el contrario se busca relevar la pluralidad de matices que construyen un imaginario social del cual son partes los sujetos a entrevistar.

Centrarse de forma única y omnipresente en los discursos que emanan desde la modalidad EMTP, sería asumir que ella emerge y se instaura en la sociedad de forma espontánea y aislada a otros discursos, esto sería incurrir en otro error vital para esta propuesta metodológica, ya que son las diferencias y objeciones las que permiten dar fondo y forma al imaginario de la EMTP. Los criterios para la selección de los sujetos son variados, pero siempre deben mantener una tangente que una con la EMTP:

- Jóvenes de EMTP, ya que básicamente es esta la modalidad en la que se enmarca los estudiantes de la especialidad. Se busca intencionar las entrevistas en aquellos sujetos 
que en hubiesen podido optar por ambas (formación humanista o EMTP) alternativas en algún momento de la elección.

- Jóvenes de ambos géneros, buscando la paridad y la equidad de género, asumiendo con ello la importancia que tienen la equidad de género y la empleabilidad de la mujer en la actividad turística.

- $\quad$ Estudiantes del INSAT Valdivia de la especialidad de turismo.

- Informantes claves tales como: apoderados, profesores y empresarios vinculados. Los cuales tienen una participación implícita en la configuración del imaginario social, materializando una serie de prácticas y códigos.

- $\quad$ Autoridades y agentes del sistema político.

Considerando los criterios anteriores, los sujetos a entrevistar son los siguientes:

- 15 alumnos de EMTP del INSAT, Valdivia.( $3^{\circ}$ medio especialidad Turismo). De los cuales 8 son mujeres y 7 son hombres.

- 3 Profesores de EMTP, del INSAT, Valdivia. (Especialidad Turismo)

- 3 Apoderados

- 4 Sujetos del ámbito productivo

- 1 Sujeto de representatividad de las políticas públicas.

\section{Estructura de las entrevistas:}

Para ella existe una estructura en la cual, si bien no es rígida, permite espacios para los discursos y voces emergentes, contiene directrices que permiten tener lineamientos que conduzcan a la coherencia con los objetivos del estudio y permitan desarrollar un análisis adecuado del modelo EMTP, y es por este motivo que se opta por una entrevista semi-estructurada, con preguntas definidas, según área de pertenencia y sujetos que se entrevistará, las cuales serán recogidas en una pauta determinada.

\section{Análisis de las entrevistas:}

A partir del llamado "giro lingüístico" de la década de los 60 y que según numerosos autores, comenzó dicho giro a comienzos del siglo XIX, cuando efectivamente el lenguaje pasa a ser objeto de estudio en múltiples disciplinas. Es a partir de este hecho que se realiza un nuevo tratamiento metodológico a los procesos discursivos con la construcción de nuevas formas de análisis, planteando maneras alternativas a las metodologías clásicas de carácter positivistas. Buscando por sobre todo caer en reducciones y positivismos que 
aproximen a un análisis cualitativo de calidad.

El abordaje del análisis de las entrevistas busca salvar las dificultades metodológicas que se exponen anteriormente y que, por medio de las posibles soluciones que se desprenden del abordaje metodológico, se busca la aportación y generación de conocimientos emergentes y coherentes para la investigación.

Considerando lo anterior, el análisis de las entrevistas, supone las siguientes etapas:

\section{A. Etapa "0" agrupación de discursiva.}

Según los objetivos específicos y la producción discursiva de los sujetos a entrevistar, se identifican ciertos nudos discursivos, los cuales están preestablecidos, lo cual no indica que ante la emergencia de nuevos nudos estos no sean incluidos a posteriori, esta agrupación será realizada en coherencia a las áreas de estructuración de las entrevistas, pudiendo establecer las siguientes agrupaciones basales.

- Elección modalidad.

- Elección especialización.

- Motivaciones y expectativas.

- Proyecciones y campo laboral.

- Futuro laboral.

- Identidad del yo.

De forma emergente es posible que nazcan nuevas agrupaciones descolgadas desde el discurso de las partes.

\section{B. Etapa "inicio" Análisis del discurso}

Es importante recordar que, lo que los sujetos dicen es el resultado de un proceso más largo de análisis y se anexa de mejor forma con el discurso que con su origen y a su vez, da cuenta que aquello que puede ser dicho bajo contextos determinados y enmarcado en un discurso situacional.

\section{Etapa "Cruce" Análisis del discurso, cruce de participantes de la modalidad}

Dentro del análisis del discurso y la construcción del imaginario social se hace necesario contrastar la información que surge de la observación y las entrevistas, buscar referencias en los agentes políticos y sociales que en ella participan, como a la vez con los interventores del proceso. 


\section{Resultados Preliminares}

Tomando en consideración el estudio de caso, enmarcado en el desarrollo de la tesis para acceder al grado de magister y considerando que estos son resultados preliminares, podemos observar algunos puntos relevantes a la hora de construir el imaginario de un grupo de alumnos de $3^{\circ}$ medio con respecto a las motivaciones, interventores en la elección, expectativas y proyecciones asociadas a su proceso de formación en un liceo de la modalidad EMTP.

\section{Elección modalidad:}

Un alto número de los estudiantes entrevistados señala que una de sus principales motivaciones para elegir esta modalidad tienen relación con la opción de "algo con que contar al cabo de la enseñanza media", popularmente conocido como el "cartón", ante lo cual Jaziel 17 años, menciona, "el liceo nos ayuda a no quedar con una mano adelante y otra atrás al momento de salir". De aseveraciones como esta se desprende que el $90 \%$ de entrevistados que asocian su elección de la modalidad por el título, no obstante, a lo mencionando anteriormente destaca un alto porcentaje, cercano al $40 \%$ que sumen su elección a la baja dificultad del modelo EMTP, Deyanira 17 años "cuando me cambie lo hice sabiendo que no tendría ni química ni física, que son los ramos más difíciles de la media", ante ello se declara creer o asumir que esta modalidad es menos complicada y exigente, asumiendo esta aseveración por la no existencia dentro de la malla las asignaturas de física y/o química, y como consecuencia de ello esperan tener muchos de ellos un mejor promedio.

Dentro de las respuesta y/o aseveraciones múltiples vinculadas al análisis del discurso de los actores de la modalidad, destaca, el que aproximadamente 3 de cada 10 estudiantes eligieron la modalidad por desconocimiento o no haberse informado de otras opciones, Nicolás 16 años "no tuve mayor información de donde ir o que seguir estudiando, elegí este liceo casi a principio de año cuando las clases ya estaban por empezar", sin embargo, de los estudiantes entrevistados se señala que 1/4 lo selecciona por vocación el interés hacia el área de acción de la especialidad, como primera opción, esta misma variable se repite en todos los entrevistados ya sea en segundo, tercero y/o cuarto lugar.

\section{Interventores en la decisión:}

Ante las conversaciones que se desprende de las entrevistas en profundidad realizadas a los alumnos, asociada a los agentes interventores en su decisión, con respecto a la elección de la modalidad EMTP, la totalidad de entrevistados indica que siempre paso por ellos la decisión; lo cual no quiere decir que no hubo otros agentes que 
apoyaran, criticaran o condujeran la elección; pero no se reconoce la influencia directa de los interventores en la decisión, Francisca 17 años "fui yo quien elegí el colegio, pasó principalmente porque a mí me gustaba de antes...mis papas me apoyan en todas mis decisiones" Rocio 17 años "mis papas no se meten mucho en mis decisiones, mientras tenga buenas notas les da lo mismo el colegio", sin embargo, se observa que si bien son ellos lo que escogen lo hacen en un alto porcentaje cerca del $90 \%$ pensada el grupo familiar con una batería de motivos asociados, los cuales van desde seguir al hermano y/o familiar o bien por recomendación de los padres, e incluso 3 de cada 10 reconoce que lo hace pensando en amigos o ex compañeros de colegio.

Un problema latente, que se pudo observar en los procesos de selección, a los cuales se someten los alumnos previo al ingreso a los liceos de EMTP, dice relación con los problemas de conducta mostrados por los estudiantes que derivó en el arribo de alguno de ellos en estos liceos, cerca del $15 \%$ dice haber llegado a este tipo de colegios por problemas de conducta o conflictos con otros compañeros en sus colegios de origen. "me cambie del liceo anterior por problemas con un compañero y su grupo de amigos y para evitar peleas después y ataos con sus amigos me vine al INSAT"

\section{Expectativas, Proyecciones y campo laboral:}

Cuando se conversa y dialoga sobre el futuro, en especial con adolecentes, se hace necesario acotarlo a un plazo determinado de tiempo, para ello, y en todo momento se les habló de un periodo de uno o dos años, como máximo, una vez terminado sus estudios medios, paralelamente se identificó hitos asociados a estas expectativas y proyecciones de futuro agrupadas y definidas en relación al mundo laboral, académico, social y familiar.

Los resultados fueron diversos y complejos, observándose en ellos variadas visualizaciones y conceptualizaciones del futuro cercano de los estudiantes y sus padres. Esto se plasma $100 \%$ de los entrevistados (estudiantes) ve su futuro post estudios en la modalidad de EMTP con trabajo estable, pero lo particular es que se ven trabajando, ya sea dentro o fuera del área, Francisca 17 años "me gustaría hacer mi práctica en el Parque Oncol y quedarme trabajando a lo que termine y agarrar experiencia para después", también se puede relevar de este ítem que 2 de cada 10 estudiantes se ven con trabajo en áreas totalmente distintas a las que imparte el Liceo EMTP, como menciona Diego 17 años "no me veo trabajando en turismo, como dicen mis viejos (papas), creo que es muy difícil encontrar trabajo en turismo y creo que voy a trabajar en algo como lo de mi papá...". Adjunto a ello, se cree en la totalidad de los entrevistados en la fácil inserción laboral asociada a la EMTP. 
Por otra parte 5 de cada 10 estudiantes creen (ya sea como $2^{\circ}$ o $3^{\circ}$ opción) en la posibilidad de generar su "Negocio Propio", ante ello el empleo autogestionado nace como las oportunidades más rentables dentro de sus posibilidades, pero les aparece como oportunidad en un futuro ideal una vez terminado sus estudios y con alguna experiencia laboral. Finalmente, dentro de las posibilidades de considerar la continuación de estudios superiores, las menciones desde la conversación derivadas de los estudiantes, son bajas se aprecia que 2 de cada 10 estudiantes piensan en la factibilidad de realizar un Pre-U y de ellos el $50 \%$ piensa en estudiar turismo como primera o única opción es decir que 1 de cada 10 estudiantes tiene deseos de continuidad de estudios superiores en el área de especialización del liceo de EMTP.

\section{Afirmaciones relevantes}

En el análisis del discurso y la construcción del imaginario social de los estudiantes de turismo de la modalidad EMTP (estudio de caso INSAT) se pueden observar algunas afirmaciones y cifras relevantes, entre ellas:

El 100\% de los entrevistados dicen estar mejor preparados para el mundo laboral, esto se ve confirmado al preguntar, que piensan sus padres con respecto a la modalidad del liceo donde sus hijos estudian, ante ello la totalidad cree tener más y mejores herramientas para la vida laboral. Sin duda esta apreciación se ve reforzada con la calificación que le ponen al colegio "liceo" donde estudias, se observa que si bien esta es alta 6,0 de promedio, queda un punto abajo con respecto a la especialidad que eligieron, que alcanza un 6,9. Para la totalidad de los alumnos existe una valoración altísima de la especialidad y de cómo esta supero sus expectativas, incluso en aquellos que llegaron al "liceo" por descarte y por la misma circunstancia a la especialidad.

Por otra parte y en la vereda opuesta 5 de cada 10 de los estudiantes dicen que no les mostraron otras opciones y la modalidad EMTP fue la única opción que barajaban ante ello ni colegios de origen ni padres les mostraron otras modalidades educativas, y en la misma línea 8 de cada 10 alumnos declararán no haber recibido en $8^{\circ}$ básico alguna orientación o recomendación con respecto donde cursar estudios secundarios ni mucho menos con respecto a su vocación.

\section{Conclusiones.}

Basadas en los avances de la investigación y en los resultados preliminares obtenidos, estas se pueden agrupar en 3 aristas de trascendencia, asociados a los fines de la investigación: 
Motivación: Esta está dada principalmente por el hecho de salir con un "titulo" y no quedar sin herramientas como ellos mismos mencionan, también se observa en este estudio, que ellos "saben", por sus fuentes de información (estudiantes del liceo y/o amigos) que esta modalidad de enseñanza se aleja de las matemáticas o las ciencias básicas, por lo cual, la ven como un modelo de educación más abordable. Finalmente para el estudio en el ámbito de la motivación también aparece como relevante el hecho de la información previa que se tiene del establecimiento de EMTP. Les ayuda a tomar la decisión trascendente en un momento de cambios "vitales" como lo es la adolescencia, finalmente y al contrario que se podría pensar se declara que la motivación de la lección está pensada más en el "cartón" como paso o situación de logro que por los réditos económicos del mismo.

Agentes intervinientes en la elección: Para los alumnos entrevistados, en su mayoría aseguran haber tomados la decisión por ellos mismo y que no influyen directamente los padres o amigos en esta decisión, pero sin embargo, se valora altamente la opinión de los padres o del grupo familiar en el afán de contribuir con esta elección a una "mejora" social y económica del grupo. Paralelamente se menciona lo importante que son las visitas que se realizan por parte de alumnos y representantes del establecimiento de EMTP a sus colegios de origen.

Expectativas y proyecciones: Si bien en la mayoría se observa y describe el deseo de perfeccionamiento o bien continuar estudios. Tan sólo cerca del 30\% declara querer continuar estudios asociados a la profesión a la cual le conduce la modalidad y especialidad, la universidad no se encuentra es su horizonte cercano.

Al hablar de proyecciones y escenario ideal, 6 de cada 10 entrevistados dice soñar o idealizar las ideas de emprender con su negocio propio, ya sea o no relacionado con la especialidad y finalmente dentro de esta construcción de imaginario se busca casi como mantra de vida el mejorar las condiciones de vida de su grupo familiar y el de ellos, pero no sólo desde la arista económica, sino que también de áreas sociales y relacionales.

\section{Bibliografía}

Blas, F. d., \& Planells (2009, pág. 160). Retos actuales de la Educación TécnicoProfesional. Madrid: Fundación Santillana.

Brunner, J. (2000). Globalización y Futuro de la Educación: Tendencias, Desafios y Estrategia: Seminario sobre Prospectivas de la Educación en América Latina y el Caribe. Santiago, Chile 123-156

Castro, E., y Orellana, W. (2010). La Educación Media Técnico Profesional en Chile: Entre la Desarticulación y La Indefinición. Osorno, Chile: Edit. Intersecciones Educativas U. de los Lagos.

Castoriadis, Cornelius (2004). Sujeto y verdad, Buenos Aires: FCE. 
Castoriadis, C. (1983). La institución imaginaria de la sociedad. Vol. 1. Barcelona: Tusquets

Durkheim, E. (2000). Educación y Sociología. Mexico DF:Edit. Colofon.

Feldman, R. (2006). Psicología con Aplicaciones en Países de Habla Hispana. Mexico DF: Edit. McGraw-Hill / Interamericana Editores, S.A. de C.V.

Foucault, M. (2005, pág. 39). La arqueología del Saber. Buenos Aires, Argentina: Siglo Veintiuno.

Miranda, M. (2003). Transformación de la Educación Media Técnico-Profesional, Políticas Educacionales en el Cambio de Siglo. La Reforma del Sistema Escolar de Chile. Santiago de Chile: Edit. Editorial Universitaria.

Sepúlveda, L., Ugalde, P., \& Campos, F. (2008). La Enseñanza Media técnico profesional en Chile: Orientaciones desde la perspectiva de sus actores. Santiago: Centro de Investigación y desarrollo de la Educación, CIDE Universidad Alberto Hurtado.

Véliz, C. (1965). Obstáculos Para el Cambio en América Latina. Santiago de Chile: Edit. Universitaria.

Pino Batory, Martín (2003). "Nuestra Cultura Tecnológica, Desde sus Orígenes Hasta Fines del siglo XIX". Santiago de Chile: Edit. Editorial Universidad.

Kohlberg, L. (1984) The Psychology of Moral Development: Essays on Moral Development. San Francisco USA: Vol. 2.Edit. Harper \& Row. 
